\title{
Conversations autour du plurilinguisme : théorisation du pluriel et pouvoir des langues
}

\author{
Danièle Moore \\ daniele_moore@sfu.ca
}

SIMON FRASER UNIVERSITY

\begin{abstract}
Résumé
Tandis qu'on note depuis quelques années une floraison de concepts cherchant à mieux théoriser la complexité et le pluriel en didactique des langues, ces nouveaux termes ne servent-ils qu'à dépoussiérer ou remettre à la mode des notions antérieures ? Entrent-ils en conflit ou en résonance? Et comment?

Cette contribution vise à poser quelques repères au sein d'un parcours situé, subjectif, à travers quelques jalons qui marquent la théorisation de la compétence plurilingue et pluri-/interculturelle (CPP) en Europe, notamment francophone. On discutera comment ces conceptualisations du plurilinguisme entrent en écho avec un autre concept du champ aujourd'hui largement circulant, celui de translanguaging. La contribution invite à resituer quelques espaces-temps du dialogue académique et invoque le pouvoir des langues dans la complexification conceptuelle.

Mots-clés : plurilinguisme, compétence plurilingue et pluriculturelle, translanguaging, didactique du plurilinguisme
\end{abstract}

\begin{abstract}
A number of concepts have emerged in recent years in an attempt to better theorize complexity and pluralism in language education. What do these new terms add to previous notions, and how do they resonate with each other?

This contribution traces a subjective trajectory through the theorization of plurilingual and pluri-/intercultural competence (PPC), focusing on Francophone Europe. It examines how these various conceptualizations of plurilingualism resonate with those of translanguaging, which are broadly circulating in the field. This contribution situates this academic dialogue in time and space, and invokes the power of languages in academic conceptual complexity.
\end{abstract}

Key words: plurilinguism, plurilingual and pluricultural competence, translanguaging, plurilingual education 


\section{Théoriser le pluriel, petite histoire située}

Cette contribution vise à poser quelques repères au sein d'un parcours situé, subjectif, à travers quelques jalons qui marquent la théorisation de la compétence plurilingue et pluri-/ interculturelle (CPP) en Europe francophone. Ce paysage théorique et épistémologique va nous amener à tracer des topographies mobiles à l'intérieur même des conversations qui se sont construites, plus particulièrement à partir des années 1990, autour des concepts de plurilinguisme et de compétence plurilingue, en espérant que ceux-ci permettront de mieux situer ces notions par rapport à une autre notion aujourd'hui largement circulante, celle de translanguaging (García et Wei, 2014). On y évoque le pouvoir des langues dans la théorisation du pluriel, en examinant comment le dialogue plurilingue encourage des mises en miroir conceptuelles susceptibles de soutenir la complexification de la réflexion intellectuelle. La publication plurilingue d'un ouvrage clef du domaine, Le Précis du plurilinguisme et du pluriculturalisme (Zarate, Levy et Kramsch, 2008), sert d'exemple pour illustrer quelques-uns de ces feuilletés réflexifs.

Ces concepts portent des idéaux autour du fait plurilingue. Ils portent aussi, intrinsèquement, une idée de dynamique, de circulations, de mobilité. Avait-on besoin de nouveaux concepts, finalement, pour décrire des phénomènes sociaux vieux comme le monde humain? Ces concepts sont-ils vraiment nouveaux ? Et enfin, faut-il les traduire?

Une mise en circulation terminologique n'est pas anodine. Choisir d'introduire un nouveau concept dans un champ de réflexion ne peut que s'accompagner d'une réflexion critique. Lorsque Coste met en circulation en 1991 le concept de compétence plurilingue, il s'agit d'une idée «qu'il a frottée à d'autres », s'inspirant de travaux aussi bien européens que nord-américains pour repenser la compétence en langue des apprenants et les intrications subtiles de leurs répertoires déjà-là et en devenir (Coste et Hébrard, 1991; Coste, de Pietro et Moore, 2012). Largement inspiré de la sociolinguistique du contact et de la migration, aux travaux de l'école suisse autour de Py et ses collègues (Gajo, Matthey, Moore et Serra, 2004; Grosjean, 1982; Lüdi et Py, 1984; Py, 2007) et ceux de l'école grenobloise autour de Dabène (1994) et de Billiez (1998), on interroge, par exemple, les maillages et méchages de langues, les variantes de contact et les marques transcodiques, dans les apports subtils qu'ils peuvent conférer à la conscientisation des processus d'appropriation pour les apprenants. Ces marques sont définies comme l'ensemble des phénomènes révélateurs du contact de langues et de cultures, des traces et d'expressions sémiotiques multiples, autant mimogestuelles que linguistiques ou vestimentaires, tous les néocodages ou énoncés métalinguistiques, les infractions aux normes phonologiques ou syntaxiques, les procédés d'ajustement et de collaboration, le travail de formulation et de figuration, etc. Toute trace qui 
témoigne, autrement dit, dans l'interparole et du point de vue des locuteurs eux-mêmes, de la rencontre au sein d'un événement langagier de langues différentes et des représentations que portent les locuteurs sur celles-ci, sur les contraintes situationnelles et sur la signification sociale qu'ils leur attribuent (voir de Pietro, 1988 et Heller, 2002). Ces atouts des plurilingues (Moore et Gajo, 2009; Herdina et Jessner, 2002) constituent pour eux des « boîtes à outils » pour communiquer et apprendre, notamment sous l'angle des connaissances métalinguistiques, qui soutiennent le développement des compétences transversales, transférables d'une langue à d' autres : « Ainsi, l'atout plurilingue (le savoir métalinguistique chez les plurilingues) est actualisé de façon interlinguistique, de telle sorte que les pratiques d'appui, de passage ou de mise en relation constituent un lieu d'observation privilégié de cet atout» (Bono, 2006, p. 42). Mais il s'agit aussi de développer une vision plus humaniste des compétences et de l'apprentissage et une «manière d'être » dans ses langues (Beacco, 2005).

Qualifié de « renversement copernicien » (Blanchet, Calvet, et Robillard, 2007), de «coup de force», de «putsch didactique» (Blanchet et Chardenet, 2015; Conteh et Meyer, 2014; Coste, 2008) et de «pavé dans la mare» (Castellotti et Moore, 2015), le concept de CPP s'impose rapidement comme un réel «changement paradigmatique» pour la didactique des langues et du plurilinguisme (Piccardo et Puozzo, 2015). Il s'agit ici de changer radicalement les manières de concevoir les compétences ainsi que les objectifs et les normes d'apprentissage, de dénativiser et désidéaliser le locuteur idéal natif, de réinstaurer le social et la personne au centre des processus d'appropriation.

Prendre au sérieux le plurilinguisme appelle, ainsi, à changer en profondeur nos manières de conceptualiser les relations entre les langues, et les relations entre les locuteurs et leurs langues; en d'autres termes, à tisser les liens entre pratiques, représentations et contexte(s) (Castellotti (dir.), 2001; Castellotti et Moore, 2015; Moore, 2001). Or, les représentations du «bon plurilingue » évoluent au fil de l'histoire et selon les contextes. De la tour de Babel à ses confettis (Calvet et Calvet, 2013), en passant par les notes de terrain de Champollion ${ }^{1}$ déchiffrant, en 1824 , en deux langues et trois systèmes d'écriture la Pierre de Rosette, ou encore Rabelais (1532) pour qui être plurilingue, c'est être un citoyen éduqué : « [il faut] que tu apprennes les langues parfaitement. Premièrement, la Grecque ... . Secondement, la Latine. Et puis l'Hébraïcque ..., et la Chaldaïcque \& Arabicque pareillement» (p. [41] $)^{2}$,

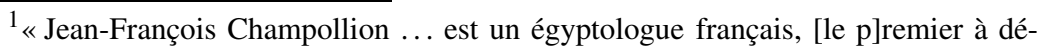
chiffrer les hiéroglyphes, Champollion est considéré comme le père de l'égyptologie » (Wikipedia, s.d., « Champollion»).

${ }^{2}$ Viennent seulement ensuite dans cette liste les arts, la géométrie, l'arithmétique et la musique.
} 
les exemples de pratiques plurilingues font partie de notre monde. Mais en France aujourd' hui, la langue arabe est-elle encore une langue qu' on choisit de mettre en avant pour exhiber socialement son éducation comme au temps de Rabelais? On voit ici comment se brossent à grands traits des paysages situés du plurilinguisme, qui s'ancrent dans des histoires, elles-mêmes situées. Il est ainsi fondamental de comprendre le développement des concepts par rapport à l'histoire, mais aussi par rapport à des histoires qui sont celles de personnes, de voyages et de rencontres. On attrape des idées parce que nous sommes des personnes avec nos propres histoires. Et cela est aussi un point important que cette contribution s'essaye à illustrer.

\section{Trajectoires et rencontres, comment les idées s'entre-frottent}

«Les concepts voyagent et il vaut mieux qu'ils voyagent en sachant qu'ils voyagent» (Morin, 1990, p. 154). Cette métaphore de la trajectoire empruntée à Edgar Morin est le fil d'Ariane que nous tirons ici. Les concepts se déplacent, voyagent et quand ils reviennent, ils ne sont pas exactement les mêmes. Nos histoires, vécues ou imaginées, influencent notre parcours et notre inscription dans un champ, dans une posture, ici par rapport au plurilinguisme. Poser un regard réflexif sur nos trajectoires vécues et pensées participe d'une démarche d'engagement dont on ne peut faire l'économie. Pour ma part, mon histoire et celle de ma famille s'inscrit dans les Alpes françaises, aux frontières de la Suisse et de l'Italie. Ma mère, née en 1920, et mon frère, né en 1940, ne parlaient pas français quand ils sont arrivés à l'école à l'âge de 7 ans. Ils étaient savoyards, locuteurs du franco-provençal. Ma mère n'a pourtant jamais su qu'elle parlait le franco-provençal, et mon frère l'a appris au détour d'une conversation il n'y a pas si longtemps. On leur a toujours dit, et ils ont toujours cru, qu'ils parlaient « le patois ». Langues non nommées, sans légitimité, minorisées. Mon père, lui, était locuteur de l'italien, langue de l'immigration alors dévalorisée en France, et je ne l'ai découvert qu'à 12 ans lors d'un voyage au Val d'Aoste pour rencontrer cette branche de la famille. Je n'avais jamais entendu mon père parler italien auparavant. Pourtant, ce n'est pas une langue que mes parents ont souhaité que j' apprenne à l'école. J'ai appris d'abord l'anglais à partir de 12 ans, puis l'allemand en deuxième langue (alors même que tous deux, résistants pendant la Deuxième Guerre mondiale, et ma mère fille de poilu blessé à Verdun pendant la Première, détestaient cette langue qui portait pour eux trop de blessures). On se déplace ainsi avec et à travers des imaginaires, qu'on traine parfois avec nous, des valises d'histoires avec lesquelles on voyage dans une Histoire qui nous dépasse le plus souvent. Et puis, ces valises ne sont parfois pas ouvertes, des petites choses y sont rangées que parfois nous n'avons pas laissé échapper, ou qu'on ne connait pas nécessairement. 


\section{Des familles à l'école : le plurilinguisme, un champ, une lentille, une posture}

Cette image de la mobilité, de la trajectoire, de la ligne de vie, de la traversée de paysages et de topographies me permet d'insister sur le fait que notre champ d'étude, le plurilinguisme, est fondamentalement interdisciplinaire. On y joue de différentes focales grossissant ou floutant plus ou moins différents aspects, qu'ils soient plutôt socio- ou psycholinguistiques, qu'ils relèvent de l'anthropologie, de l'ethnographie de la communication, de l'acquisition des langues secondes et tierces, des bilinguismes, des études sur la migration, sur les créoles, ou sur la revitalisation des langues, de la politique linguistique, de l'éducation aux langues ou bien encore, de l'éducation bilingue.

Mon intérêt premier est de comprendre comment les locuteurs apprenants plurilingues font usage de leurs ressources plurielles en famille, à l'école et dans les autres contextes de leurs apprentissages. C'est à partir de l'observation de ces pratiques ordinaires que la conceptualisation de la Compétence plurilingue et pluriculturelle (CPP) se développe; puis, dans un effort de mise en continuité critique des actions d'intervention, se forme la Didactique du plurilinguisme, qui regroupe les différents scénarios d'apprentissage utilisant des approches multilingues (Candelier, 2008; Candelier et Castellotti, 2013). Il s'agit ainsi du maillage de trois fils, entrelaçant les interrelations entre :

(i) des interactions plurilingues et les questions d'appropriation dans des environnements plurilingues et multiculturels pour

(ii) informer les politiques des langues et

(iii) imaginer, enfin, de nouvelles formes d'interventions éducatives, contextuelles, pour préparer les apprenants et les enseignants à vivre dans des sociétés linguistiquement et culturellement diverses.

Le social y est toujours présent, articulant les rapports entre les individus et les contextes dans lesquels ils évoluent et au sein desquels ils façonnent, et se façonnent, leurs répertoires et ressources plurilingues (Conteh et Meier, 2014).

La conceptualisation de la Compétence plurilingue se construit donc comme un changement paradigmatique, où l'acteur se situe au centre du questionnement, questionnement qui se fonde sur une théorie sociale et une pratique ethnographique (Hymes, 1984) prenant en compte les attitudes, les croyances et les valeurs dans leurs intrications et leurs interdépendances avec des contextes. Pour Coste, Moore et Zarate (1997), alors,

Parler de compétence plurilingue et pluri-/ interculturelle c'est ... s'intéresser à la compétence à communiquer d'acteurs sociaux en mesure d'opérer dans des langues et des cultures différentes, de jouer des rôles d'intermédiaires, de médiateurs linguistiques et culturels, à même aussi de gérer et de remodeler cette compétence plurielle au cours de leur trajectoire personnelle. (p. 129) 


\section{Pratiques et (re)présentations}

Au-delà de l'analyse des pratiques, dans et autour de la classe, il est ainsi primordial de s'intéresser aux représentations, aux croyances et aux idéologies dans lesquelles ces pratiques s'ancrent et se négocient. Quelle est notre représentation des langues? Qu'est-ce qui compte comme une langue? Quelle est notre représentation de l'éducation? Ces interrogations, tantôt d'ordre épistémologique, éthique et politique, problématisent les notions de langues, de transmission, d'appropriation et d'identité au sein d'espaces de représentations, de croyances, de normes, dans des espaces idéologiques et imaginés. Quand je dis «ma mère parlait franco-provençal», je me (re)présente aussi, c'est mon identité. Même si le franco-provençal n'est pas une langue que je parle, il fait partie de mon imaginaire linguistique.

Ainsi, si la compétence linguistique et culturelle est pour partie le produit historique de forces sociales, la compétence d'un locuteur dans plusieurs langues et cultures reste unique. Parler alors d' « acteur social plurilingue» met l'accent sur la relation entre l'action entreprise dans un contexte donné et le recours stratégique de l'acteur social concerné à différentes langues et à la pluralité, sans oublier les efforts stratégiques qu'il déploie, si nécessaires, pour dissimuler une partie de son répertoire linguistique et culturel, son aptitude à mobiliser au plus haut prix son capital symbolique d'expérience de l'altérité (Coste, Moore et Zarate, 1997, p. 30).

\section{Une posture plurilingue réflexive}

Inspiré du travail du Suisse François Grosjean (1982, mais voir aussi 2008 et 2010), on insiste sur le fait que c'est la synthèse qui caractérise la compétence des bi-plurilingues (le pluri-), plutôt que le nombre de langues impliquées (porté dans la terminologie, par le multi-). C'est alors pour insister sur la synthèse (le tout, l'ensemble et les effets de reconstructions induits) et sur la posture plurilingue adoptée sur les phénomènes observés (Moore et Gajo, 2009), qu'on choisit d'introduire une nouvelle terminologie afin de montrer que si on parle des mêmes phénomènes, le regard porté sur ceux-ci est autre (Marshall et Moore, 2018). Cet ensemble de travaux s'inscrit en dissonance des discours circulant sur les frontières de langues et les rapports à la norme, notamment dans les projets d'apprentissage. Comme le rappellent Jessner-Schmid et Kramsch (2015) :

Multi[pluri]lingualism serves to decenter the sources of power by contesting the discourses of purity, normality and authenticity associated with monolingualism and by giving legitimacy to more hybrid forms of expression, across codes, modes and modalities. (p. 8) 
L'adoption d'une posture plurilingue réflexive est ainsi indispensable pour réfléchir les situations et en proposer des interprétations qui dépassent de simples processus d'ajustement de principes, de démarches, de contenus d'enseignement ou d'exercices à des réalités locales (Beacco et Coste, 2017; Castellotti, 2014) pour prendre au sérieux et travailler l'historicité des situations d'appropriation des langues, leur diversité, y compris « interne», du point de vue des parcours des personnes concernées, avec les enjeux politiques, sociaux, individuels qui en découlent, y compris subjectivement (Coste, 2002, 2004). Ici encore s'inscrit la valeur de considérer les dynamiques circulatoires de la biographisation des expériences altéritaires dans les parcours, en incluant le/la chercheur-e dans cette contextualisation et cette historicisation (Huver, 2014). Une posture plurilingue en didactique, c'est alors créer les conditions à l'école et à ses marges pour que les personnes engagées dans cette appropriation prennent conscience de la diversité langagière pour la mobiliser réflexivement, dans et par l'expérience, dans de nouvelles rencontres (Castellotti, 2017; voir aussi Blanchet et Coste, 2010; Candelier et Schröder-Sura, 2015; North et Piccardo, 2016).

\section{Circulations des concepts dans des espaces et des langues : des mises en écho}

Les idées n'ont pas d'existence en elles-mêmes, elles dépendent de la langue dans laquelle elles sont exprimées (Liddicoat, 2018; Liddicoat et Zarate, 2009). Aller jusqu'au fond de la notion de plurilinguisme, c'est alors, aussi, s'interroger sur les jeux de traductions dans de nouveaux contextes linguistiques, nationaux, entendus comme processus spécifiques supposant que des connotations sont ajoutées ou supprimées, ce qui entraîne que l'idée elle-même est reconstruite. Le flux des idées ne peut être entendu comme une simple transplantation dans un autre contexte, car celles-ci s'inscrivent dans des réseaux existants d'idées, de théories, de langues, mais aussi de personnes, à travers un processus de mises en écho et d'adaptations qui tient compte des idées déjà-là des gens, de la structure sémiotique des débats en cours, de l'histoire. Qu'estce qu'une société est prête à recevoir comme nouvelle idée? Où peut-elle se mettre?

Revenons alors maintenant brièvement sur la notion de plurilinguisme dans la tradition française, que je connais mieux.

Pourquoi plurilinguisme(s)? Pourquoi parler de plurilinguisme, quand on avait l'habitude de parler de multilinguisme ? La notion, qui circulait déjà dans la Convention européenne de 1954 (Conseil de l'Europe, 1954), se diffuse et prend de l'ampleur notamment par le biais de la revue française de sociolinguistique que dirige Calvet, Plurilinguismes, dont le premier numéro paraît en 1999 et porte sur les villes plurilingues (les contributions réunies portent sur 
la France, l'Angleterre, des villes d'Afrique, etc.). Un autre numéro spécial de la même revue porte plus spécifiquement sur «Les emprunts». On y trouve des contributions interrogeant les phénomènes d'alternance codique, ou codeswitching, dans les pratiques ordinaires des plurilingues, mais on y trouve aussi une contribution sur la question des alternances d'appropriation dans la classe (Moore et Py, 1995). La contribution emprunte aux travaux sociolinguistiques suisses (comme par exemple Grosjean, 1982; Lüdi et Py, 1986) et, en didactique des langues, à celui de Dabène (1994), considérant que si on adopte une posture plurilingue pour l'analyse du répertoire des locuteurs et des apprenants, la notion d'emprunt est inutile car le répertoire du plurilingue ne fait qu'un.

Tandis que peu avant, un séminaire réunit à Londres un certain nombre des mêmes collègues sur des questions avant tout sociolinguistiques concernant l'alternance (Milroy et Muysken, 1995), notamment autour des parlers bilingues des migrants (Dabène et Moore, 1995), un colloque à orientation sociolinguistique et didactique est organisé en 1995 par Coste au CRÉDIF (Centre de recherche pour l'enseignement et la diffusion du français). Consacré à la thématique des Alternances de langues, il réunit en conférences pléniaires Heller, Lüdi, Obadia, Poplack et van Lier. Plusieurs publications découlent de ces rencontres. On trouve ainsi un numéro spécial de la revue Plurilinguismes portant plutôt sur les perspectives sociolinguistiques de la rencontre des langues (Deprez, 1995, p. 1). Deux numéros complémentaires interrogent plus spécifiquement les alternances de langues comme levier d'appropriation, en particulier dans les situations d'enseignement. Le premier est un numéro des Études linguistique appliquée (ELA, 108), intitulé «Alternances des langues et apprentissages » (Castellotti et Moore, 1997). Le second est un numéro des Cahiers du français contemporains, intitulé «Alternances des langues et construction de savoirs " (Moore et Castellotti, 1999; mais voir aussi Castellotti, 2000, 2001; Castellotti (dir.), 2001). Il ne s'agit ainsi pas seulement de remettre en question la notion socialement construite de frontière de langue, mais de porter un regard holistique sur l'ensemble des systèmes sémiotiques qui servent la communication.

On distingue ainsi nettement des travaux qui relèvent de la sphère politique, où le plurilinguisme mais aussi la compétence plurilingue relèvent peu à peu d'une doxa, et les travaux qui s'inscrivent dans la sphère académique où ces notions sont affinées, complexifiées et très tôt remises en question parce que les deux sphères se superposant brouillent, et parfois effacent, les débats intellectuels qui continuent à surgir autour de ces notions (Castellotti, 2010; Coste, 2010; Moore et Castellotti, 2008). 


\section{Plurilinguisme et translanguaging}

En cherchant à situer des questionnements autour du plurilinguisme et du translanguaging au sein du petit itinéraire historique jusque-là tracé, on rappellera qu'on attribue en règle générale la notion de translanguaging (trawsieithu en gallois) aux travaux de Williams (1994) portant sur les questions d'alternance d'enseignement-apprentissage dans l'enseignement immersif bilingue au Pays de Galles : «What is translanguaging and how can it be used? The simplest definition is : receiving information in one language and then using it in the other language » (Williams, 2002, p. 47), précisant encore que «the way in which bilingualism is used actively is translanguaging, which reinforces the interrelationship between the two languages while also reinforcing the languages $\gg$ (p. 46).

Ces alternances d'enseignement-appropriation, d'abord décrites sous le terme de translanguifying évoluent, par le biais des conversations entre Williams, Whittal, Baker, et Lewis, vers celle de translanguaging, qui paraît mieux traduire en anglais le concept gallois (Williams, Lewis et Baker, 1996; Lewis, Jones et Baker, 2012). On voit dans cet exemple de glissement terminologique qu'un concept nouveau trop abscons n'est pas efficace. On a besoin aussi de simplicité dans la complexité. De chaque côté, on trouve abondamment cités les travaux de Cummins (1981), comme ceux de Canale (1981) et Canale et Swain (1981) ou encore ceux de Jacobson (1990). Williams (1996) écrit ainsi :

A theory of bilingual teaching, developed in the United States by Jacobson and Faltis, is based on cues for switching languages when teaching. But there are sixteen different cues. It is unreasonable to expect any teacher to be aware of such a high number of cues when he/she is teaching. By switching the emphasis to a consideration of the linguistic and conceptual demands made on the child in every classroom situation, these cues can be reduced to 5 ways in which language is used in teaching and in developing concepts .... (p. 51)

Vingt ans plus tard, l'idée d'alternance didactique entre les langues est cependant abandonnée par les auteurs prônant l'adoption du translanguaging, qui oublient trop rapidement que la notion s'est développée initialement dans un effort de scénarisation des tissages d'appropriation à l'école, inspirée justement du travail des sociolinguistes qui ont permis de réinvestir les pratiques ordinaires des plurilingues comme fondamentalement riches, originales, complexes, polyglossiques et polyphoniques, et de nature à favoriser le développement conceptuel et l'appropriation; et ce faisant, de questionner la question de la norme à l'école. Par exemple, García et Lin (2016) rappellent que :

Translanguaging ... posits the linguistic behavior of bilinguals as being always heteroglossic (see Bailey 2007; Bakhtin 1981), always dynamic, responding not to two monolingualisms in one, but to one integrated linguistic system. It 
is precisely because translanguaging takes up this heteroglossic and dynamic perspective centered on the linguistic use of bilingual speakers themselves, rather than starting from the perspective of named languages (usually national or state languages), that it is a much more useful theory for bilingual education than code-switching. (p. 3)

Bien que les auteures stipulent que le translanguaging ressemble au codemixing/switching mais que le terme translanguaging engage le chercheur dans une vision fluide et dynamique du langage, la définition qu'elles en proposent n'est, on le voit, pas sans entretenir de forts échos avec les travaux précédemment cités sur le plurilinguisme et les marques transcodiques et la vision holistique et dynamique du langage dans lesquels ces travaux s'inscrivent. Les travaux francophones défendent en effet de la même façon que les langues sont des constructions sociales (Calvet, 1999), soumises à des normes historiquement situées au sein de dynamiques écologiques particulières (Calvet et Calvet, 2013), que les plurilingues naviguent des dynamiques plurielles les situant de manière particulière au sein de ces écologies (Grosjean, 1982), que leur répertoire est unique et intégré (Dabène, 1994) et que les alternances jouent des rôles dans les processus identitaires et d'appropriation (Lüdi et Py, 1986). Partant de la classe pour certains, de la rue pour les autres (Lewis, Jones et Baker, 2012), des phénomènes observables similaires sont analysés selon des focales différentes, selon des sensibilités et des contextes qui s'éloignent de plus en plus les uns des autres. Pourtant, l'idéal porté reste le même : reconnaître la compétence des plurilingues et favoriser l'appui sur leurs pratiques pour aller plus loin.

\section{Plurilinguisme, translanguaging, language awareness : les lieux au carrefour des idées}

On voit ainsi que d'un côté, on s'est intéressé à l'enseignement bilingue dans des situations minorées, dans des situations où les langues sont territoriales, comme l'anglais et le gallois, au pays de Galle. Du côté français et suisse, les interrogations des sociolinguistes et didacticiens du plurilinguisme portent surtout alors sur l'enseignement de ce qu'on appelait à l'époque les Langues et cultures d'origine. On s'intéresse alors par exemple à l'enseignement bilingue avec une langue de migration, comme le français et l'arabe. Surtout, on lance un débat et des recherches sur la question du renouvellement didactique qu'appelle une posture plurilingue sur l'enseignement des langues, et sur l'atout d'appropriation que peuvent soutenir des démarches plurielles d'ouverture aux langues pour tous (Candelier, 2003, 2008; Moore, 1995; Perregaux, de Goumoëns, Jeannot et de Pietro, 2003). Si on situe, à titre d'exemple, Bangor (au Pays de Galle) comme un lieu clef d'effervescence d'idées, on voit d'un côté se développer toute une réflexion sur les pratiques d'alternances de 
langues en classe (le translanguaging), tandis que dans les mêmes années, s'y déroule la première conférence de Language Awareness organisée par James et Garrett où Hawkins est invité d'honneur (mais aussi des linguistes réputés comme David Crystal, etc.). Une association est créée, l'International Association for Language Awareness, ainsi qu'une revue, Language Awareness Journal. Le premier volume est publié en 1992. On trouve rassemblés dans le comité éditorial John Sinclair, Gillian Donmall, Peter Garrett, Paolo Balboni, Christopher Brumfit, Michel Candelier, Margaret Early, Eric Hawkins, Carl James, Mike Scott, David Singleton, John Trim (dont on retrouvera l'influence dans les travaux du Conseil de l'Europe), et puis Leo van Lier. Des rencontres, des notions en circulations et en questions... Ces travaux entrent en écho les uns avec les autres, les uns s'intéressant davantage aux potentialités cognitives particulières que favorise le plurilinguisme pour les apprenants, les autres pour les moyens didactiques de les soutenir et en favoriser le développement (Cenoz, Gorter et May, 2017; Garrett et Cots, 2018). Plusieurs idées sont mises en avant : ces caractéristiques peuvent se constituer en atout d'apprentissage; elles se construisent autour d'une conscience/conscientisation de ces atouts; ces travaux n'établissent pas de distinction entre apprenant et plurilingue, considérant que tout apprenant est un plurilingue en devenir et tout plurilingue reste un apprenant (Gajo et al., 2004; Py, 2007). Ainsi que JessnerSchmid et Kramsch (2015) le résument :

In Herdina and Jessner's Dynamic Model of Multilingualism (2002) metalinguistic awareness or multilingual awareness has been identified as an emergent core factor of multilingual proficiency and is currently under investigation in an increasing number of studies on multilingual learning and teaching. (Jessner, 2006; Moore, 2006; De Angelis \& Otwinowska 2014). (p. 4)

Le Language Awareness essaime rapidement en dehors du Royaume Uni, notamment chez les Grenoblois : L'éveil aux langues (Dabène et Billiez, 1990), puis chez les Suisses l'Éducation et ouverture aux langues à l'école ou EOLE (Balsinger, Köhler, de Pietro et Perregaux, 2012; Perregaux et al., 2003), déjà influencés par les travaux de Roulet (1980), mais aussi au Canada, sous l'appellation l'Education interculturelle et diversité linguistique ou ELODIL (Armand et Maraillet, 2013). En France encore, Le Portfolio européen des langues (conçu par Castellotti, Coste, Moore et Tagliante en 2004) propose une section intitulée «Diversité des langues », où les activités proposées (comme le recueil photographié des panneaux plurilingues dans la ville pour porter un regard critique sur la place des langues dans la société) portent de claires références aux activités proposées par Hawkins et Donmall à la suite des travaux de ce dernier sur le Language Awareness en Angleterre (Castellotti et Moore, 2005; Hawkins, 1984; voir aussi Dagenais, Moore et Sabatier, 2013). 
Ces derniers travaux se réclament de la didactique du plurilinguisme (Moore, 2006), des approches plurielles en didactique (Candelier et SchröderSura, 2015), ou encore de l'Éducation plurilingue et interculturelle (Beacco et Coste, 2017), orientations didactiques qui elles-mêmes s'appuient sur la volonté de développer chez les apprenants cette fameuse Compétence plurilingue et pluriculturelle (Moore et Castellotti, 2008; North et Piccardo, 2016), dont il est intéressant de noter la concomitance du développement avec celle du translanguaging, les deux termes apparaissant dans la littérature consacrée la même année :

By a curious coincidence, 1996 is also the year in which the term 'translanguaging' is recorded (in relation to bilingual teaching in Wales). Translanguaging is an action undertaken by plurilingual persons, where more than one language may be involved. (Council of Europe, 2018, p. 28)

Ainsi, si certains concepts fleurissent plus ou moins en même temps et semblent se développer dans des lieux séparés, ils émergent, de fait, de conversations entre des personnes qui s'entre-connaissent et partagent des idéaux et des postures autour du plurilinguisme et de l'éducation aux langues. Ces concepts s'essaiment ensuite de manières différentes selon les priorités et les besoins locaux. Ainsi, le paysage épistémologique de la compétence plurilingue rassemble parmi les auteurs cités Bourdieu, Cummins, Dabène, Grosjean, Hamers, Hawkins, Kramsch, Lüdi, Martin-Jones, Porcher, Py, Romaine, Skutnabb-Kangas, mais aussi Hymes, Gumperz, et Goffman (Coste, de Pietro et Moore, 2012). Les Européens sont ainsi très inspirés aussi par les chercheurs d'Amérique du nord. On voit aussi immédiatement se profiler un intérêt central pour les personnes et leurs trajectoires dans les langues. Ainsi, Zarate intègre à l'étude de référence pour le Conseil de l'Europe les dialogues biographiques, autour des histoires de vie et de langues de Matias, Wolfgang, Maria, Albert, Martine et les autres (Coste, Moore et Zarate, 1997). Surtout aussi, la compétence plurilingue cherche à théoriser un répertoire intégré, une vision holistique de la compétence, sous-tendue par une thématisation du déséquilibre et la remise en cause du locuteur idéal, du natif, de la maîtrise de la langue, du modèle du monolingue sans identité (voir, par exemple, Martinez, Moore et Spaëth, 2008). On s'intéresse au fait que cette compétence plurilingue est un atout pour une conscience métalinguistique enrichie. On s'intéresse aux interactions translinguistiques, aux capacités de médiation, de ponts, de passages. On s'intéresse aux liens entre les biographies, les histoires de vie, les trajectoires d'apprentissage et les processus de mise en relation des langues.

Le concept fait carrière et s' autonomise assez rapidement de ses auteurs. Il devient une politique, et prend dès lors une forme idéologique. Alors qu'à l'origine, c'est une nouvelle notion qui veut faire bouger (Coste, 2004), qui remet en question des représentations et des idéologies de ce qu'est le bi- le pluri-, qui 
s'inscrit en réaction aux conceptions du locuteur idéal et à l'exclusion chomskyenne des dimensions socioculturelles dans l'apprentissage. Qui insiste sur la nature située, le caractère social de la compétence. Qui prend appui sur des études de terrain des théories alternatives du développement bi-plurilingue. Qui met l'accent sur la complexité des dynamiques interactionnelles et resitue les objectifs et les interrelations entre langues et apprentissages à l'école (Bigot, Bretegnier et Vasseur, 2013; Stratilaki, 2005).

Ces exemples montrent l'importance de considérer le développement des concepts en synchronie, mais aussi en diachronie, de s'intéresser aux supports éditoriaux et aux institutions qui en portent la diffusion, tout en réfléchissant aux potentialités que chaque langue ouvre pour la (re)conceptualisation.

\section{Conversations réflexives plurilingues : un exemple entre français, anglais et chinois}

Tout ce travail de réflexivité inter et translinguistique trouve une expression toute particulière dans l'aventure éditoriale que constitue l'écriture du Précis du plurilinguisme et du pluriculturalisme (Zarate, Lévy et Kramsch, 2008 [en français], 2011 [en anglais], 2016 [en chinois]). L'ouvrage, en rassemblant plus de 90 contributeurs issus de 68 institutions dans 20 pays différents (en Europe, Amérique du nord, Afrique et Asie) travaillant ensemble au travers d'un éventail de langues (souvent une troisième ou quatrième langue commune), interroge, dans sa conception même, la circulation internationale des idées et les «embûches linguistiques, culturelles et disciplinaires » $(\mathrm{Fu}, 2017$, p. 183) liées à une telle entreprise.

Sa traduction en chinois pose tout un nouveau jeu de dialogues pour créer des zones passerelles et passer entre les (histoires de) langues (voir Zarate, Gohard-Radenkovic et Fu [傅荣, 2015). L'équipe chinoise a l' avantage de pouvoir s'appuyer et sur la version française et sur la version anglaise du Précis pour passer au chinois. Elle s'est déjà frottée à la traduction du Cadre européen (CECR) en chinois et à l'introduction de nouveaux concepts, comme celui de compétence plurilingue. Ainsi, tandis que la publication en anglais du Précis entraîne le changement du titre de l'ouvrage qui de Précis du plurilinguisme et du pluriculturalisme, devient (sous la pression éditoriale), Handbook of Multilingualism and Multiculturalism, en chinois en revanche, l'équipe de traducteurs choisit de s'aligner sur les conceptions propres au Précis et propose pour la traduction du titre de l'ouvrage : Introduction aux idées de la pluralité linguistique et culturelle (多元语言和多元文化教育思想引论 [duōyuán yǔyán hé duōyuán wénhuà jiàoyù sīxiăng yǐn lùn]). Parler d' «idées » de la pluralité, terme qui signifie ici littéralement en chinois "pensées et réflexions », paraît le choix le plus pertinent pour l'équipe sinophone. D'abord, parce qu'il désigne le plurilinguisme et le pluriculturalisme en tant que notion, concept 
ou visions sur la langue et la culture et la langue-culture (les deux préfixes «multi- » et «pluri- » sont souvent traduits sans distinction par 多 $(d u \bar{o})$, qui veut dire «nombreux »). Ensuite, de cette façon, parce que le titre en chinois est « concis, clair et facile à lire et à retenir » (Fu, 2017, p. 187). Pernet-Liu et Dai (2009) précisent ainsi :

Le multilinguisme implique deux notions : d'un côté, il renvoie à la connaissance d'un certain nombre de langues donc à la compétence individuelle, traduite par多语言能力[duō yǔyán nénglì]; de l'autre côté, il s'agit de la coexistence de langues différentes dans une société donnée, donc d'un phénomène social, traduit par 多语言化 [duō yǔyán huà]. Le plurilinguisme, lui, vise à mettre en valeur la construction d'une compétence communicative d'un individu « à laquelle contribuent toute connaissance et toute expérience des langues et dans laquelle les langues sont en corrélation et interagissent» (CECR, 13). Ainsi, ce terme est traduit par 多元语言能力 [duōyuán yǔyán nénglì] ou 语言多元化 [yǔyán duōyuán huà]. 多元 [duōyuán], en plus de l'aspect quantitatif, exprime surtout l'existence de langues différentes, diverses et multiples. La traduction joue aussi avec la place de 多元 [duōyuán], situé avant ou après le mot 语言 ([yǔyán] langue), et 语言多元化 [yǔyán duōyuán huà] insiste aussi sur l'esprit ouvert d'un individu pour tolérer et accepter la langue et la culture d'autrui. D'autre part, le choix du suffixe 化 [huà] en chinois pour traduire «-isme », plutôt que le suffixe 主义 [zhǔyì] en usage habituellement mais moins dynamique, renvoie à un champ en pleine évolution, un processus en cours amenant au but ciblé. La construction des mots en chinois permet d'avoir aussi 多语言能力([duō yǔyán nénglì]; compétence plurilingue) ou多语言思想[duō yǔyán sīxiăng]; littéralement : «pensée du plurilinguisme »; c'est ainsi que le titre du Précis est traduit). (p. 202)

La traduction de représentation sociale, qui fait l'objet du chapitre 6 (intitulé «Discours sur les langues et représentations sociales ») coordonnée par Moore et Py (2008), présente un autre exemple de fin travail de mise à plat conceptuelle au moment de la traduction. Kramsch choisit un mode bilingue pour questionner la notion dans son Contrepoint au chapitre : elle s'y adresse tour à tour en français «Au lecteur francophone» puis en anglais « To the Anglophone reader » pour discuter d'une «notion qui ne se traduit pas ». Se référant aussi bien aux travaux de Bourdieu qu'à ceux de Foucault ou de Fairclough et de Gee, elle écrit ainsi : «My first reaction as an American language teacher is to wonder how I managed to do without a notion as useful as that of représentation sociale in thinking about second language acquisition in multilingual contexts. But I am not sure to understand what it really means ». Pernet-Liu et Dai (2009) soulignent des difficultés supplémentaires au moment de passer au chinois :

Les hésitations des traducteurs sont le reflet de l'usage varié que les différents auteurs du Précis ont fait de cette notion : il s'agit parfois d'une référence très forte à la notion de «représentation sociale » ancrée dans les travaux de 
Moscovici, comme on la trouve sous la plume des auteurs des introductions de ces [deux] chapitres; ou bien il s'agit d'un usage plus ou moins flou du mot désignant d'une manière fluctuante une opinion, une idée, une conception, une manière d'imaginer ..., avec une référence implicite et plus ou moins lointaine à l'histoire de cette notion. Cette instabilité de l'usage reflète l'histoire riche du succès d'une notion transdiplinaire (Stratilaki, 2011 : 31-82) qui continue de marquer les sciences humaines dans des réinterprétations diverses. En chinois, il n'y pas un seul mot qui prendrait des valeurs différentes. S'il s'agit de «représentation » au sens flou, il y a plusieurs expressions possibles, en particulier 表现 [biăoxiàn], 代表 [dàibiǎo], 概念 [gàiniàn]. Pour parler des représentations sociales dans un sens académique, le mot 社会表征 [shèhuì biăozhēng] a été introduit en chinois grâce à la traduction du livre de Moscovici Les représentations sociales en 2011. Finalement les traducteurs ont la plupart du temps choisi (意识表征 [yìshi biǎozhēng]) ; caractérisation de conscience) pour englober à la fois la dimension d'image (表征 [biăozhēng] ; signe) et la dimension mentale (意识 [yìshi] : conscience). La version chinoise désigne toute expression qui révèle la conscience et l'esprit d'un sujet. (p. 212)

On voit ici le minutieux effort de (re)conceptualisation et de prise de distance critique qu'implique tout travail de traduction et combien ce tâtonnement peut nous apporter en termes de réflexion/réflexivité sur les concepts que nous faisons nôtres.

\section{Conversations plurilingues autour du plurilinguisme, pour un kaléidoscope de la complexité}

Cette contribution a voulu insister sur le fait que, d'une part, les concepts sont portés par des langues qui portent en elles des possibilités. Il est important de ne pas en faire l'économie, pour au contraire s'appuyer sur les formes de réflexion que permettent les creux conceptuels. L'anglais porte des dynamiques que le français ne permet pas. Comment en effet traduire un concept si économiquement efficace que translanguaging pour insister sur le mouvant et le mobile. Le passage par d'autres langues encore apporte d'autres formes de subtilités, met à jour des univers de références situés, des intraduisibles, des mises en ombre ou en lumière et, tel un kaléidoscope, les fait briller en « réfléchissant » les différences et la diversité.

On retiendra ainsi surtout que les concepts sont portés par des femmes et des hommes qui ont leur propre histoire, aussi de langues. Si les concepts se développent dans des contextes socio-historiquement marqués, ils sont d'abord le fruit de rencontres humaines; les concepts sont lus, compris et repris dans des contextes socio-historiquement situés et dans des langues particulières. Ils s'inscrivent en écho ou en dissonance dans des discours déjà circulants et il est nécessaire de comprendre ces temporalités, ces spécificités et les enjeux de pouvoir dans lesquels ils s'inscrivent, qui les poussent et les repoussent. Ce sont ces rencontres et frottements qui font avancer la pensée humaine et c'est 
dans la force du dialogue et dans les intraduisibles, parfois les incompréhensibles, que se forment les idées nouvelles. C'est ainsi par les rencontres, dans des espaces-temps-langues situés, qu'on frotte ensemble nos idées, parce que, comme le rappelle Castellotti (2014) :

La recherche en DDL [didactologie-didactique des langues] ne peut trouver un avenir qu'en pensant, fondamentalement, la diversité humaine constitutive des situations d'appropriation. Cela implique de s'intéresser d'abord aux parcours de ceux qui s'y engagent, aux relations qui les caractérisent, au statut que, en tant que chercheur-e-s, nous pouvons leur attribuer à travers les interprétations que nous en faisons et qui sont aussi tributaires de nos parcours et des relations que nous instaurons par nos implications dans ces situations et par notre positionnement vis-à-vis des institutions. (p. 123)

\section{Références}

Armand, F. et Maraillet, E. (2013). Éducation interculturelle et diversité linguistique (Élodil). Montréal : Université de Montréal. www.elodil.umontreal.ca/guides/ education-interculturelle-et-diversite-linguistique

Balsinger, C., Köhler, D.B., de Pietro, J.-F. et Perregaux, C. (2012). Éveil aux langues et approches plurielles : de la formation des enseignants aux pratiques de classe. Paris : L'Harmattan.

Beacco, J.-Cl. (2005). Langues et répertoire de langues : le plurilinguisme comme «manière d'être " en Europe, Étude de référence pour le Guide pour l'élaboration des politiques linguistiques éducatives en Europe. Strasbourg : Conseil de l'Europe. www.academia.edu/36080364/Langues_et_répertoires_de_langues_ le_plurilinguisme_comme_manière_d_être_en_Europe

Beacco, J.-Cl. et Coste, D. (dir.). (2017). L'Éducation plurilingue et interculturelle : la perspective du Conseil de l'Europe. Paris : Didier.

Bigot, V., Bretegnier, A. et Vasseur, M. (dir.). (2013). Vers le plurilinguisme? Vingt après. Paris : Éditions des archives contemporaines.

Billiez, J. (dir.). (1998). De la didactique des langues à la didactique du plurilinguisme : hommage à Louise Dabène. Grenoble : Centre de didactique des langues (CDL) - Linguistique et didactique des langues étrangères et maternelles (LIDILEM).

Blanchet, Ph., Calvet, L.-J. et Robillard, D. (2007). Carnets d'Atelier de Sociolinguistique [numéro thématique : Un siècle après le Cours de Saussure : la linguistique en question], 1 .

Blanchet, Ph. et Chardenet, P. (dir.). (2015). Guide pour la recherche en didactique des langues et cultures : approches contextualisées. Paris : Publications de l'Agence universitaire de la francophonie (AUF) - Collectif Inter-Réseaux en Didactique des Langues (CIRDL). 
Blanchet, Ph. et Coste, D. (dir.). (2010). Regards critiques sur la notion d'" interculturalité » : pour une didactique de la pluralité linguistique et culturelle. Paris : L'Harmattan.

Bono, M. (2006). La compétence plurilingue vue par les apprenants d'une L3 : le plurilinguisme est-t-il toujours un atout ? Éducation et sociétés plurilingues, 20, 39-50.

Calvet, L.-J. (1999). Pour une écologie des langues du monde. Paris : Plon.

Calvet, L.-J. et Calvet, A. (2013). Les confettis de Babel : diversité linguistique et politique des langues. Paris: Organisation internationale de la francophonie.

Calvet, L.-J. (dir.) (1999). Plurilinguisme, 1. CITED ON ms.pg.8. Journal name NOT FOUND via Google.

Canale, M. (1981). From communicative competence to communicative language pedagogy. Dans J. Richards et R. Schmidt (dir.), Language and communication (p. 227). New York : Longman.

Canale, M. et Swain, M. (1981). A theoretical framework for communicative competence. Dans A. Palmer, P. Groot et G. Trosper (dir.), The construct validation of test of communicative competence (p. 31-36). Washington, DC : Teaching English to Speakers of Other Languages (TESOL).

Candelier, M. (2008). Approches plurielles, didactiques du plurilinguisme : le même et l'autre. Recherches en didactique des langues et des cultures : les Cahiers de l'Acedle [numéro thématique : L'Alsace au cour du plurilinguisme], 5, 65-90.

Candelier, M. et Castellotti, V. (2013). Didactique(s) du/des plurilinguisme(s). Dans J. Simonin et S. Wharton (dir.), Sociolinguistique des langues en contact, modèles, théories. Dictionnaire encyclopédique des termes et concepts (p. 293-318). Lyon : École normale supérieure (ENS) Éditions.

Candelier, M. et Schröder-Sura, A. (2015). Les approches plurielles et le CARAP : origines, évolutions, perspectives. Babylonia [numéro thématique : Les approches plurielles des langues et des cultures], 2, 12-19.

Candelier, M. (dir.) (2003). L'éveil aux langues à l'école primaire: Evlang, bilan d'une innovation européenne. Bruxelles, Belgique : De Boek-Duculot.

Castellotti, V. (2000). Alterner les langues pour construire des savoirs bilingues. Le Français dans le Monde : Recherches et applications [numéro thématique : Actualité de l'enseignement bilingue], janvier, 118-124.

Castellotti, V. (2001). La langue maternelle en classe de langue étrangère. Paris : CLE International.

Castellotti, V. (2014). Contexte, contextualisation, cultures éducatives. Quels usages? Pour quelles orientations de la recherche en DDL? Dans S. Babault, M. Bento, L. Le Ferrec et W. Spaëth (coord.), Actes du colloque international Contexte global, contextes locaux : tensions, convergences et enjeux en didactique des langues (p. 111-124). Paris : Fédération internationale des professeurs de français. fipf.org/sites/fipf.org/files/actes_colloque_contexte_global_et_contextes_locaux_sorbonne_ nouvelle_paris_3_2014.pdf 
Castellotti, V. (2017). Pour une didactique de l'appropriation : diversité, compréhension, relation. Paris : Didier.

Castellotti, V., Coste, D., Moore, D. et Tagliante, Ch. (2004). Portfolio européen des langues : collège. Paris : Didier/Conseil de l'Europe.

Castellotti, V. et Moore, D. (2005). Les portfolios européens des langues : des outils plurilingues pour une culture éducative partagée. Repères [numéro thématique : Français et langues étrangères et régionales à l'école : quelles interactions ?], 29, 167-183.

Castellotti, V. et Moore, D. (2015). La compétence plurilingue et pluriculturelle. Genèses et évolutions. Dans Ph. Blanchet et P. Chardenet (dir.), Guide pour la recherche en didactique des langues et cultures : approches contextualisées (p. 291302). Paris : Éditions des archives contemporaines.

Castellotti, V. (dir.) (2001). D’une langue à d'autres, pratiques et représentations. Rouen : Presses universitaires de Rouen.

Castellotti, V. (dir.). (2010). Recherches en didactique des langues et des cultures : les Cahiers de l'Acedle [numéro thématique : Notions en questions - les plurilinguismes], 7.

Castellotti, V. et Moore, D. (dir.). (1997) Études de Linguistique Appliquée [numéro thématique : Alternances des langues et apprentissages], 108.

Cenoz, J., Gorter, D. et May, S. (dir.). (2017). Encyclopedia of language and education : Language awareness and multilingualism ( $3 \mathrm{e}$ éd.). Berlin : Springer.

Champollion, J.-F. (s.d.). Extrait tiré du Wikipédia. fr.wikipedia.org/wiki/Jean-Fran\%C3 \%A7ois_Champollion

Conseil de l'Europe. (1954). La Convention culturelle européenne (Paris, 1954). www. coe.int/fr/web/conventions/full-list/-/conventions/rms/090000168006458c

Council of Europe. (2018). Common European Framework of Reference for Languages : Learning, teaching, assessment. Companion volume with new descriptors. Strasbourg : Council of Europe.

rm.coe.int/cefr-companion-volume-with-new-descriptors-2018/1680787989

Conteh, J. et Meier, G. (dir.). (2014). The multilingual turn in languages education : Opportunities and challenges. Bristol, UK : Multilingual Matters.

Coste, D. (1991). Diversifier, certes? Le Français dans le Monde : Recherches et applications [numéro thématique : Vers le plurilinguisme? École et politique linguistique], février-mars, 170-176.

Coste, D. (2002). Compétence à communiquer et compétence plurilingue. Notions en Questions [numéro thématique : La notion de compétence en langue], 6, 115-123.

Coste, D. (2004). De quelques déplacements opérés en didactique des langues par la notion de compétence plurilingue. Dans A. Auchlin, M. Burger, L. Filliettaz, A. Grobet, J. Moeschler, L. Perrin, C. Rossari et L. de Saussure (dir.). Structures et discours : mélanges offerts à Eddy Roulet (p. 67-85). Québec : Nota Bene. 
Coste, D. (2008). Éducation plurilingue et langue de scolarisation. Recherches en didactique des langues et des cultures : les Cahiers de l'Acedle, 5, 91-107.

Coste, D. (2010). Diversité des plurilinguismes et formes de l'éducation plurilingue et interculturelle. Recherches en didactique des langues et des cultures : les Cahiers de l'Acedle [numéro thématique : Notions en questions - les plurilinguismes], 7, $1-19$. journals.openedition.org/rdlc/2031

Coste, D., de Pietro, J.-F. et Moore, D. (2012). Hymes et le palimpseste de la compétence de communication. Tours, détours et retours en didactique des langues. Langage et Société, 139, 103-123.

Coste, D. et Hébrard, J. (dir.). (1991). Le Français dans le Monde : Recherches et applications [numéro thématique : Vers le plurilinguisme? École et politique linguistique], février-mars.

Coste, D., Moore, D. et Zarate, G. (1997). Compétence plurilingue et pluriculturelle : Plurilingual and pluricultural competence. Strasbourg : Conseil de l'Europe.

Cummins, J. (1981). The role of primary language development in promoting educational success for language minority students. Dans California State Department of Education (dir.), Schooling and minority language students : A theoretical framework. Los Angeles : California State University, Evaluation, Dissemination and Assessment Center. www.learnalberta.ca/content/eslapb/documents/lcebergs_ common_language.pdf

Dabène, L. (1994). Repères sociolinguistiques pour l'enseignement des langues : Les situations plurilingues. Paris : Hachette.

Dabène, L. et Billiez, J. (1990). Lidil [numéro thématique : Les langues et cultures des populations migrantes: Un défi à l'école française, 2.

Dabène, L. et Moore, D. (1995). Bilingual speech of migrant people. Dans L. Milroy et P. Muysken (dir.), One speaker, two languages : Cross-disciplinary perspectives on code-switching (p. 17-44). Cambridge : Cambridge University Press.

Dagenais, D., Moore, D. et Sabatier, C. (2013). Paysages linguistiques, plurilinguismes et contextualisations didactiques. Dans C. Cortier et M. Cavalli (dir.), Langues régionales/minoritaires dans l'éducation bi-/plurilingue : langues d'ici, langues d'ailleurs (p.23-28 ). Graz : Éditions du Conseil de l'Europe, Centre européen pour les langues vivantes. www.ecml.at/Portals/1/documents/ECML-resources/ebp-ici-FR. pdf?ver=2018-03-20-115007-950

De Pietro, J.-F. (1988). Vers une typologie des situations de contacts linguistiques. Langage et société, $43,65-89$.

Deprez, C. (1995). L'emprunt, la trace et la marque, le passage. Plurilinguismes, 9-10, $1-6$.

$\mathrm{Fu}$, R. (2017). Quelques enseignements et réflexions tirés de notre expérience de traduction du français au chinois du Précis du Plurilinguisme et du 
Pluriculturalisme (PPP). Synergies Chine [numéro thématique : La traduction : enjeux pour l'apprentissage de langues, la formation de traducteurs, la circulation internationale du savoir et des idées], 12, 183-192. docplayer.fr/135796631-You-yi-yi--duo-yuan-yu-yan-he-duo-yuan-wen-hua-jiao-yu-si-xiangyin-lun--yin-fa-de-si-kao.html

Gajo, L., Matthey, M., Moore, D. et Serra, C. (dir.). (2004). Un parcours au contact des langues : Textes de Bernard Py commentés. Paris : Didier.

Gajo, L. et Steffen, G. (2014). Sciences et plurilinguisme : savoirs et perspectives en tension. Dans A.-C. Berthoud et M. Burger (dir.), Repenser le rôle des pratiques langagières dans la constitution des espaces sociaux contemporains, (p. 107-124). Bruxelles, Belgique : DeBoeck.

García, O., et Lin, A. (2017). Translanguaging in bilingual education. Dans S. May (dir. de série), Encyclopedia of language and education: Vol. 5, O. García, A. Lin et S. May (dirs. du volume), Bilingual and multilingual education (pp. 117-130). Dordrecht, The Netherlands : Springer.

García, O. et Wei, L. (2014). Translanguaging : Language, bilingualism and education. Basingstoke, UK : Palgrave Macmillan.

Garrett, P. et Cots, J. (dir.). (2018). The Routledge handbook of language awareness. New York : Routledge.

Grosjean, F. (1982). Life with two languages. Cambridge, MA : Harvard University Press.

Grosjean, F. (2008). Studying bilinguals. Oxford : Oxford University Press.

Grosjean, F. (2010). Bilingual : Life and reality. Cambridge, MA : Harvard University Press.

Hawkins, E. (1994). Awareness of language : An introduction. Cambridge, UK : Cambridge University Press.

Heller, M. (2002). Éléments d'une sociolinguistique critique. Paris : Didier.

Herdina, P. et Jessner, U. (2002). A dynamic model of multilingualism : Perspectives of change in psycholinguistics. Clevedon, UK : Multilingual Matters.

Huver, E. (2014). Les inaccessibles de l'évaluation en langue(s) : Impensé ? Impasse ? Ferments ? Glottopol, 23, 77-100.

Hymes, D. (1984). Vers la compétence de communication. Paris : Didier.

Jacobson, R. (1990). Allocating two languages as a key feature of a bilingual methodology. Dans R. Jacobson et C. Faltis (dir.), Language distribution issues in bilingual schooling (p. 3-17). Clevedon, UK : Multilingual Matters.

Jessner-Schmid, U. et Kramsch, C. (2015). The multilingual challenge : Crossdisciplinary perspectives. Berlin : Walter de Gruyter.

Lewis, G., Jones, B. et Baker, C. (2012). Translanguaging : Origins and development from school to street and beyond. Educational Research and Evaluation, 18, 641654. doi.org/10.1080/13803611.2012.718488 
Liddicoat, A.J. (2018). Language teaching and learning as a transdisciplinary endeavour : Multilingualism and epistmological diversity. AILA Review, 31, 14-28. doi.org/10.1075/aila.00011.lid

Liddicoat, A.J. et Zarate, G. (2009). La didactique des langues face à la circulation internationale des idées. Le Français dans le monde : Recherches et applications [numéro thématique : La circulation internationale des idées], 46, 9-13.

Lüdi, G. et Py, B. (1986). Être bilingue. Berne : Peter Lang.

Marshall, S. et Moore, D. (2018). Plurilingualism amid the panoply of lingualisms : Addressing critiques and misconceptions in education. International Journal of Multilingualism, 15, 19-34. doi.org/10.1080/14790718.2016.1253699

Martinez, P., Moore, D. et Spaëth, V. (éds.) (2008). Plurilinguismes et enseignement : identités en construction. Paris : Riveneuve Éditions.

Milroy, L. et Muysken, P. (dir.). (1995). One speaker, two languages : Crossdisciplinary perspectives on code-switching. Cambridge : Cambridge University Press.

Moore, D. (2006). Plurilinguismes et école. Paris : Didier.

Moore, D. (éd.) (2001). Les représentations des langues et de leur apprentissage : références, modèles, données et méthodes. Paris : Didier-CRÉDIF-Essais.

Moore, D. (1995). Notions en questions [numéro thématique : L'Éveil du langage, 1.

Moore, D. et Castellotti, V. (2008). Perspectives de la recherche francophone autour de la notion de compétence plurilingue. Dans D. Moore et V. Castellotti (dir.), La compétence plurilingue : regards francophones (p. 11-24). Berne : Peter Lang.

Moore, D. et Castellotti, V. (dir.). (1999). Les Cahiers du français contemporain [numéro thématique : Alternances des langues et construction de savoirs], 5.

Moore, D. et Gajo, L. (dir.). (2009). International Journal of Multilingualism [numéro thématique : Plurilingualism and pluriculturalism : Francophone perspectives in education ], 6(2).

Moore, D. et Py, B. (1995). Paroles empruntée, parole appuyée ou la place des emprunts dans l'apprentissage d'une langue seconde en milieu bilingue. Plurilinguismes, 9/10, 133-145.

Moore, D. et Py, B. (2008). Introduction : discours sur les langues et représentations sociales. Dans G. Zarate, C. Kramsch et D. Lévy (dir.), Précis du plurilinguisme et du pluriculturalisme (p. 275-283). Paris : Éditions des archives contemporaines.

Morin, E. (1990). Introduction à la pensée complexe. Paris : Essai.

North, B. et Piccardo, E. (2016). Developing illustrative descriptors of aspects of mediation for the Common European Framework of Reference (CEFR) : A Council of Europe project. Language Teaching, 49, 455-459.

Perregaux, C., de Goumoëns, Cl., Jeannot, D. et de Pietro, J.-F. (dir.). (2003). Éducation au langage et ouverture aux langues à l'école (EOLE). Neuchâtel, Suisse : Secrétariat général de la CIIP. 
Pernet-Liu, A. et Dai, D. (2015). Perspectives ouvertes par la traduction chinoise du Précis du plurilinguisme et sa contextualisation sociolinguistique et didactique en Chine. Synergies Chine, 10, 199-217. gerflint.fr/Base/Chine10/pernet_liu_dai.pdf

Piccardo. E. et Puozzo, I. (dir.). (2015). Revue canadienne des langues vivantes [numéro thématique : De la didactique des langues à la didactique du plurilinguisme : un changement de paradigme possible ?, 71(4).

Py, B. (2007). Apprendre une langue et devenir bilingue : un éclairage acquisitionniste sur les contacts de langues. Journal of Language Contact, 1, 93-100.

Rabelais, F. (1532). Pantagruel. Vol. 1. Paris : Édition Nourry. fr.wikisource.org/wiki/ Pantagruel/\%C3\%89dition_Nourry,_1530

Roulet, E. (1980). Langues maternelles, langues secondes : vers une pédagogie intégrée. Paris : Hatier et le Centre de recherche et d'étude pour la diffusion du français (CREDIF).

Stratilaki, S. (2005). Vers une conception dynamique de la compétence plurilingue : quelques réflexions six ans après. Dans M.-A. Mochet, M.-J. Barbot, V. Castellotti, J.-L. Chiss, C. Develotte et D. Moore (dir.), Plurilinguisme et apprentissages : mélanges Daniel Coste (p. 155-168). Lyon : ÉNS Éditions.

Williams, C. (1994). Arfarniad o Ddulliau Dysgu ac Addysgu yng Nghyd-destun Addysg Uwchradd Ddwyieithog [An evaluation of teaching and learning methods in the context of bilingual secondary education] (thèse de doctorat inédit). Bangor, UK : University of Wales.

Williams, C. (2002). A language gained : A study of language immersion at 11-16 years of age. Bangor, UK : University of Wales Bangor, School of Education.

Williams, C., Lewis, G. et Baker, C. (1996). The language policy : Taking stock. Interpreting and apraising Gwynedd's language policy in education. Llangefni, UK : Canolfan Astudiaethau Iaith (CAI) Language Studies Centre.

Zarate, G., Gohard-Radenkovic, A. et Fu, R. (2015). Le Précis du plurilinguisme et du pluriculturalisme : une recherche internationale, face aux défis d'une conception plurilingue et d'une traduction en anglais et en chinois. Dans U. Jessner-Schmid et C. Kramsch (dir.), The multilingual challenge : Cross-disciplinary perspectives (p. 239-270). Berlin : Mouton de Gruyter.

Zarate, G., Lévy, D. et Kramsch, C. (dir.). (2008). Précis du plurilinguisme et du pluriculturalisme. Paris : Éditions des archives contemporaines. core.ac.uk/download/pdf/ 55269962.pdf

Zarate, G., Lévy, D. et Kramsch, C. (dir.). (2011). Handbook of multilingualism and multiculturalism. Paris : Éditions des archives contemporaines. (Texte d'origine publié en 2008)

Zarate, G., Lévy, D. et Kramsch, C. (dir.). (2016). 多元语言和多元文化教育思想引论 [duōyuán yǔyán hé duōyuán wénhuà jiàoyù sīxiăng yı̌n lùn= Introduction aux idées de la pluralité linguistique et culturelle]. (Trad. R. Fu). Paris : Éditions des archives contemporaines. (Texte d'origine publié en 2008) 